\title{
Understanding system-focused barriers to the identification and reporting of medication errors and adverse drug events in emergency medical services
}

\author{
Ian Howard ${ }^{1} \cdot$ Nicholas Castle $^{1} \cdot$ Loua Al Shaikh $^{1} \cdot$ Ameena Jesaimani $^{1} \cdot$ Robert Owen $^{1}$ \\ Published online: 23 April 2019 \\ (c) The Author(s) 2019
}

\begin{abstract}
Introduction Emergency medical services (EMS) represent an important and increasingly used entry point into the healthcare system. These services are often provided in the backdrop of challenging environments, with few diagnostic resources available, and for patients of varying acuity. As a result, the potential for medication errors (MEs) and adverse drug events (ADEs) is significant. However, the identification and reporting of these occurrences is poorly understood.

Methods A healthcare failure mode and effects analysis was conducted at the Hamad Medical Corporation Ambulance Service, Qatar, to identify and mitigate potential system-focused barriers to the identification and reporting of ADEs and MEs in the EMS setting. Between May 2018 and July 2018, a process map and potential failure points were developed and identified. Probabilities, severity, and hazard scores were calculated for each failure point, and action items were developed to address these. Results In total, 110 potential failure points were identified among 22 sub-processes, divided between six major processes. In terms of severity, most were classified as either 'moderate' $[n=90(80 \%)]$ or 'major' $[n=14(13 \%)]$. In terms of probability, failure points classified as 'frequent' $[n=39(35 \%)]$ and 'occasional' $[n=38(35 \%)]$ made up the majority. Through consensus, 76 failure points (69\%) were deemed necessary for action. Five strategic-level, system-focused action items were identified to mitigate against the failure points meeting the criteria for action.

Conclusions The unique circumstances that occur within the EMS setting dictate that bespoke solutions be developed to allow for ease of implementation and ongoing success of use. Strategies that also take advantage of mobile technology are crucial for a service that has mobility as one of its core functions.
\end{abstract}

\section{Key points}

The identification and reporting of medication errors and adverse drug events in the out-of-hospital emergency care setting is poorly understood.

The impact of system-based barriers to the reporting of these events is significant. This study identified 110 potential points of failure within a generic out-of-hospital medication error and adverse drug event reporting process, 76 of which warranted action.

The development of out-of-hospital specific criteria, tools and resources necessary to mitigate these potential failure points is an essential step towards reducing the burden of their burden.

Ian Howard

IHoward@hamad.qa

1 Hamad Medical Corporation Ambulance Service, Hamad Medical Corporation, P.O. Box 3050, Doha, Qatar

\section{Introduction}

Emergency medical services (EMS) represent an important and increasingly used entry point into the healthcare system. Patients transported by these services are often amongst the most critically ill or injured seeking healthcare, many of whom may require initial intervention or resuscitation prior to reaching hospital [1-7]. Given this emergent nature in which patients present to EMS, coupled with the fact that these services are often provided in the backdrop of challenging environments, with few diagnostic resources available, and for patients of varying acuity, the potential for medication errors (MEs) and adverse drug events (ADEs) is significant.

Despite this potential, little is known about the true occurrence or reporting of MEs and ADEs in the EMS setting. Current literature has largely been restricted to the identification of rates of self-reported MEs and ADEs [8-12]. A multitude of factors could potentially affect the identification and reporting of MEs and ADEs in the EMS setting, which too remain largely unknown. These range from the 
individual-specific circumstances that influence a practitioner's decision to report an ME or ADE, to the systemfocused barriers and enablers to identifying and reporting their occurrence. An understanding of these factors is crucial when encouraging practitioners and developing a system that facilitates and sustains this support.

\section{Background}

A retrospective internal audit of general drug practice was carried out over the 1-year period from January 2017 to December 2017, to better understand current practices in the use of medications within the Hamad Medical Corporation Ambulance Service (HMCAS), Qatar. In summary, 58,398 patients received 70,509 medication administrations out of 194,101 emergency patients transported for the year (30.09 patients receiving medication per 100 emergency patient transports; 36.33 drug administrations per 100 emergency patient transports). Of the patients who received a medication administration, 9230 patients received more than one medication type (4.76 patients receiving multiple medications per 100 emergency patient transports), and 6676 patients received a medication considered 'high alert/high risk' — drugs that bear a heightened risk of causing significant patient harm when they are used in error [13] (3.44 patients receiving high-risk medication per 100 emergency patient transports). Despite this, only 46 entries into the organisation's occurrencevariance-accident reporting system were found relating to medication use. Of these 45 were related to broken drug ampoules that occurred during regular operations. Zero MEs were self-reported, and one single report related to a patient experiencing an ADE was documented ( 0.0005 per 100 emergency patient transports). While the reporting of MEs and ADEs is poorly documented in the literature, the rate of reporting found within HMCAS was lower than that observed in similar studies within the EMS setting. There is evidence to support the role the health system or service plays towards the reporting of MEs and ADEs. Several system-focused barriers have been described in the general healthcare literature, including managerial-related factors regarding consequences [14-17], reporting system-related factors $[14,16]$ and managerial factors relating to motivation [17]. No literature could be identified that has similarly examined the system's influence on the identification and reporting of MEs and ADEs in the out-of-hospital emergency care setting.

As a result, a multidisciplinary team from was assembled to conduct a healthcare failure mode effects analysis (HFMEA), to better understand the system-focused factors affecting the reporting of ADEs and MEs.

\section{Methods}

The HFMEA methodology is a qualitative-based, systematic technique for risk identification and failure analysis. Developed by the US Department of Veteran Affairs National Centre for Patient Safety, the HFMEA is a prospective, multistage, multidisciplinary, modified version of the generic failure mode effects analysis technique that has been adapted for the healthcare setting. Throughout the early rounds of the HFMEA, additional input into identifying processes and potential failure points was provided by multiple alternative data sources, including clinical audit data and occurrence-variance-accident reporting system data [18, 19].

\section{Setting}

Hamad Medical Corporation Ambulance Service is the government-funded national ambulance service of Qatar, serving a population of $\approx 2.7$ million people, with a combined daily average call rate of $\approx 700$ emergency or primary community cases and 300 non-emergency or inter-facility transfers per day. The service follows a USA/UK/Australia EMS model of independently licenced emergency care practitioners for its frontline staff and operates a two-tiered system of ambulance paramedic (AP)-staffed ambulances, and critical care paramedic (CCP)-staffed fast-response vehicles.

The diverse mix of nationalities and cultures that make up the population of Qatar is echoed in the broader healthcare workforce. This has added an extra layer of complexity in the day-to-day operations of delivering healthcare in the country, given the diverse backgrounds in education and experience over and above the ethnic, cultural and language differences amongst healthcare workers in Qatar. Within the ambulance service, CCPs are recruited from EMS organizations around the world with specific training and experience in out-of-hospital emergency care. However, APs are sourced from a variety of allied healthcare backgrounds and then receive additional in-service bridging training once incountry. Given this, a large majority of EMS staff would not have had prehospital emergency care experience prior to their employment within the HMCAS. At the time of the study, no ME and/or ADE training was offered within HMCAS.

\section{Healthcare failure mode effects analysis (HFMEA) panel composition}

The primary focus for inclusion into the panel was to ensure adequate representation from all departments within the 
service that affect the reporting of ADEs and MEs, including operational staff (ground and aeromedical) and senior operations managers, as well as representatives from executive management, training, clinical audit and governance, supply chain and pharmacy. Multiple rounds of both face-to-face meetings and online correspondence occurred throughout the HFMEA process to reach the study objectives.

\section{Process and sub-process description}

For round 1, the process for identifying, reporting and investigating an ME or ADE in the EMS setting was mapped, through consensus agreement by the panel. A follow-up draft process map was re-circulated among team members for further reflection and refinement, following inclusion of the results of the internal audit data. A final draft was then independently validated by an operational CCP not involved in the process map development or audit.

\section{Hazard identification and analysis}

For round 2, potential hazards and/or points of failure were identified for each sub-process, again by team consensus. For the purposes of this study, a hazard or failure point was defined as "ways or manners in which a sub-process may potentially fail to reach and/or provide its anticipated result or outcome" [18]. The draft list was similarly supplemented by data from the audit and occurrence-variance-accident reporting system highlighted above. The updated list was then circulated among team members for independent hazard analysis and subsequent consensus agreement. Consensus was determined when $\geq 70 \%$ of participants were in agreement about assigned scores or categories throughout data collection.

For round 3, a hazard analysis was conducted on the hazards and failure points identified during round 2 . The
Table 2 Healthcare failure mode effects analysis hazard scoring matrix [15]

\begin{tabular}{|c|c|c|c|c|}
\hline \multirow[t]{2}{*}{ Probability } & \multicolumn{4}{|l|}{ Severity } \\
\hline & Catastrophic & Major & Moderate & Minor \\
\hline Frequent & 16 & 12 & 8 & 4 \\
\hline Occasional & 12 & 9 & 6 & 3 \\
\hline Uncommon & 8 & 6 & 4 & 2 \\
\hline Remote & 4 & 3 & 2 & 1 \\
\hline
\end{tabular}

HFMEA hazard analysis differs from the traditional FMEA approach in that severity and probability of failure points are assigned a category and not a numerical value (Table 1). Hazard scores were then calculated based on the assigned categories using a matrix to determine a final value (Table 2). An HFMEA decision tree was followed using the final hazard matrix scores to determine criticality, controllability and detectability of each failure point (Fig. 1).

\section{Action plan recommendations}

A fourth and final consensus round was then conducted to determine whether or not further action was required to mitigate the hazards and failure points identified as priorities, and potential action plans to achieve this. Failure points that scored $\geq 8$ following the hazard analysis were automatically included for further discussion for action (see Fig. 1 for decision tree and definitions).

\section{Institutional Review Board}

Ethical approval to conduct the study was granted by the Medical Research Centre of the Hamad Medical Corporation, Qatar.

\section{Table 1 Healthcare failure mode effects analysis severity and probability rating scale definitions [15]}

\section{Severity}

Catastrophic event

Major event

Moderate event

Minor event

Probability

Frequent

Occasional

Uncommon

Remote
Death or major permanent loss of function (sensory, motor, physiologic, or intellectual), suicide, rape, hemolytic transfusion reaction, surgery/procedure on the wrong patient or wrong body part, infant abduction or infant discharge to the wrong family

Permanent lessening of bodily functioning (sensory, motor, physiologic, or intellectual), disfigurement, surgical intervention required, increased length of stay for three or more patients, increased level of care for three or more patients

Increased length of stay or increased level of care for one or two patients

No injury nor increased length of stay nor increased level of care

Likely to occur immediately or within a short period (may happen several times in 1 year)

Probably will occur (may happen several times in 1-2 years)

Possible to occur (may happen sometime in 2-5 years)

Unlikely to occur (may happen sometime in 5-30 years) 


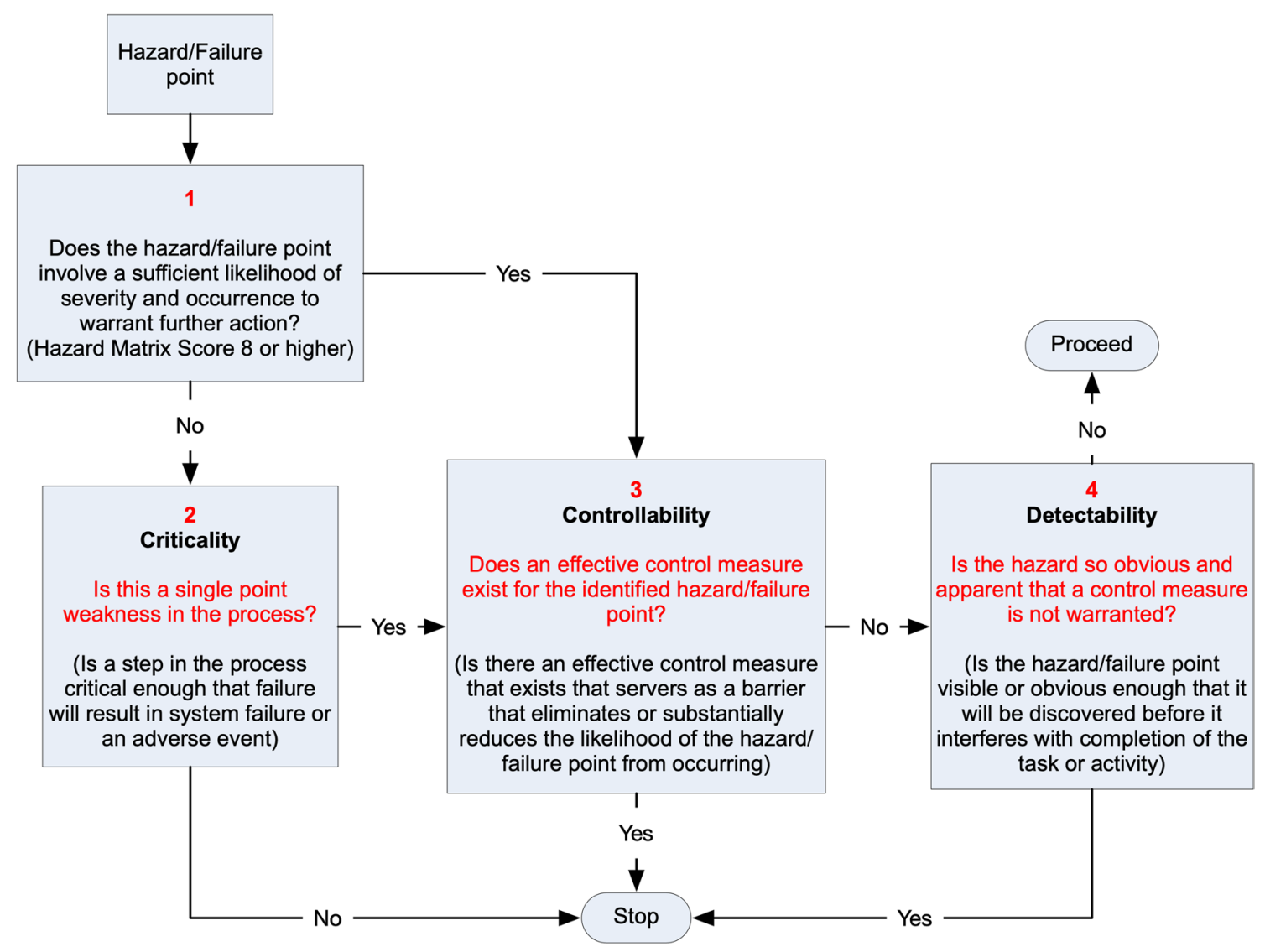

Fig. 1 HFMEA decision tree

\section{Results}

\section{Process map and hazard identification}

A final process map consisting of six major processes was produced and included the following (Fig. 2):

- Practitioner recognises ADE/ME.

- Practitioner accesses ADE/ME reporting system.

- Practitioner enters ADE/ME details into reporting system.

- Report is received, and review is conducted.

- Practitioner is provided individual feedback following review.

- Outcomes of review are compiled and shared with all practitioners for learning purposes.

Among these, 22 separate sub-processes were identified with the process "report is received, and review is conducted" process constituting the greatest number of sub-processes $(n=5)$. Altogether, 110 potential hazards/failure points were described amongst the separate sub-processes. Those based on the "report is received, and review is conducted" sub-process $[n=30(27 \%)]$ and the "practitioner accesses ADE/ME reporting system" subprocess $[n=21(19 \%)]$ constituted the greatest number.

\section{Hazard analysis}

For the 'probability' category, the failure points categorized as 'frequent' $[n=39(35 \%)]$ made up the majority, with most occurring in the "practitioner accesses ADE/ ME reporting system" sub-process $(n=14)$. This was followed by the 'occasional' probability category $[n=38$ $(35 \%)]$, the majority of which were found in the "report is received, and review is conducted" process (Table 3).

In terms of severity, failure points categorized as 'moderate' $[n=90(82 \%)]$ contributed the largest group by a significant margin. The 'major' category constituted the next largest group $[n=14(13 \%)]$, with the "practitioner is provided individual feedback following review" process the most common amongst this category. Only four hazard/failure points were categorised as 'catastrophic', divided between the "practitioner recognises ADE/ME" 


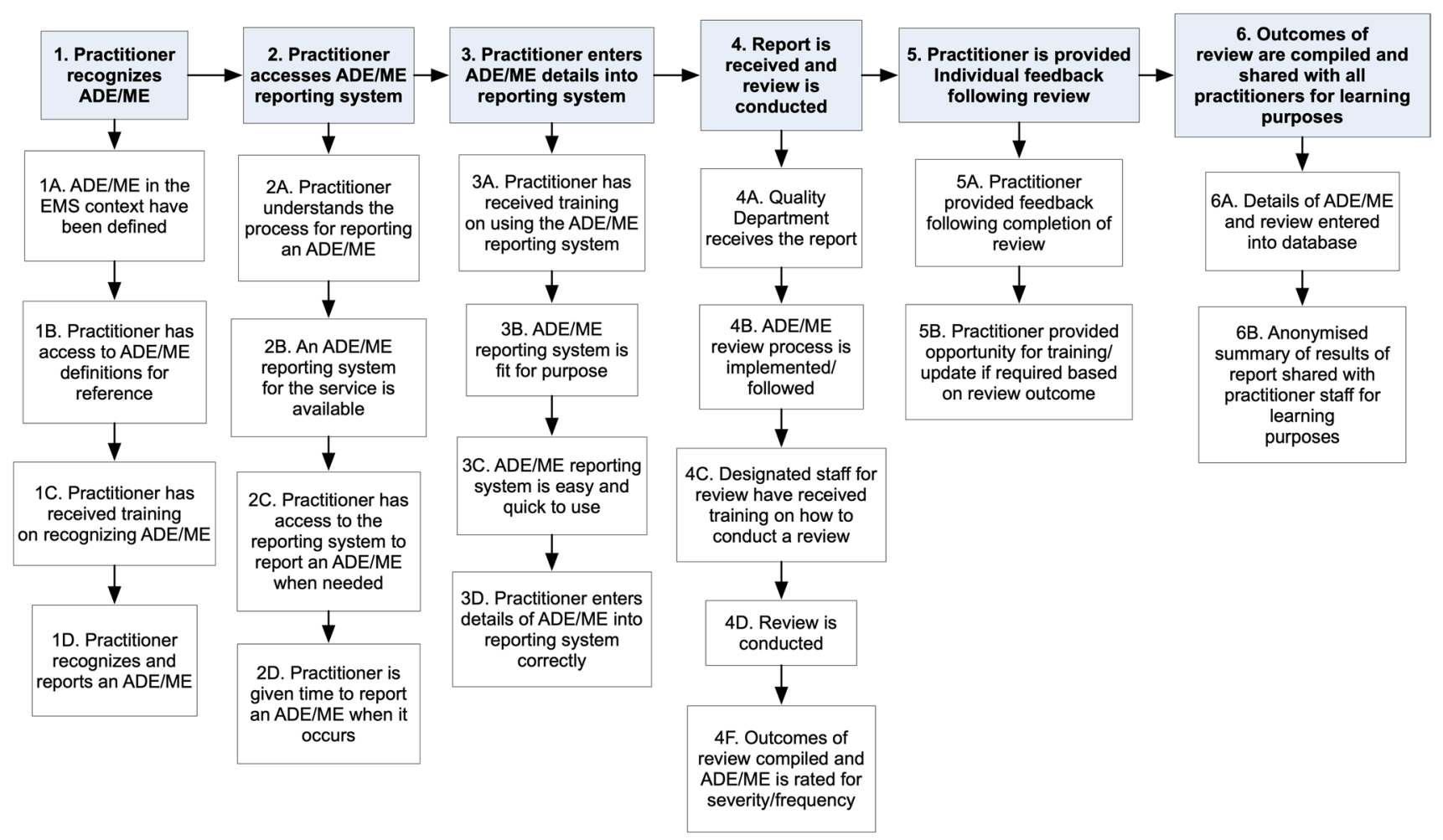

Fig. 2 Process and sub-process map

Table 3 Hazard/failure point severity and probability rating summary

\begin{tabular}{lcclll} 
Severity & \multicolumn{2}{c}{ Probability } & & & Total \\
\cline { 2 - 5 } & Frequent & Occasional & Uncommon & Remote \\
Catastrophic & $1(0.91 \%)$ & $1(0.91 \%)$ & 0 & $3(2.73 \%)$ & $4(3.64 \%)$ \\
Major & $4(3.64 \%)$ & $4(3.64 \%)$ & 0 & $5(4.55 \%)$ & $13(11.82 \%)$ \\
Moderate & $33(30 \%)$ & $32(29.1 \%)$ & $12(10.91 \%)$ & $13(11.82 \%)$ & $90(81.82 \%)$ \\
Minor & $1(0.91 \%)$ & $1(0.91 \%)$ & 0 & 0 & $2(1.82 \%)$ \\
Total & $39(34.45 \%)$ & $37(34.54 \%)$ & $12(10.91 \%)$ & $21(19.1 \%)$ & 110
\end{tabular}

sub-process $(n=1)$, "practitioner accesses ADE/ME reporting system" $(n=2)$ and "practitioner enters ADE/ ME details into reporting system" $(n=2)$ sub-processes.

\section{Action plan recommendations}

Based on the results of the hazard scoring, 44 hazard/failure points $(40 \%)$ scored $\geq 8$, thus warranting further discussion for action. Of this group, six hazard/failure points $(5 \%)$ were found to be adequately controlled for. An additional 32 hazard/failure points were deemed, through consensus, significant enough to warrant further discussion, despite a hazard score $<8$ (Table 4 ). The majority of potential failure points requiring action fell within the "report is received, and review is conducted" process $[n=20(26 \%)]$, followed by the "practitioner accesses ADE/ME reporting system" $[n=16(21 \%)]$ and "practitioner recognises ADE/ME" $[n=13(17 \%)]$ processes.

Given the objective of the study, the following systemfocused areas for improvement were identified to improve the reporting of ADEs and MEs in the EMS context:

- Action Plan 1 (AP1) Develop/implement/utilise concise, unambiguous definitions of what constitute ADEs and MEs, specific to the EMS setting. These should be readily available for reference, ideally at the point of care. Where possible, these should also be incorporated into a service's clinical practice guidelines to assist with 


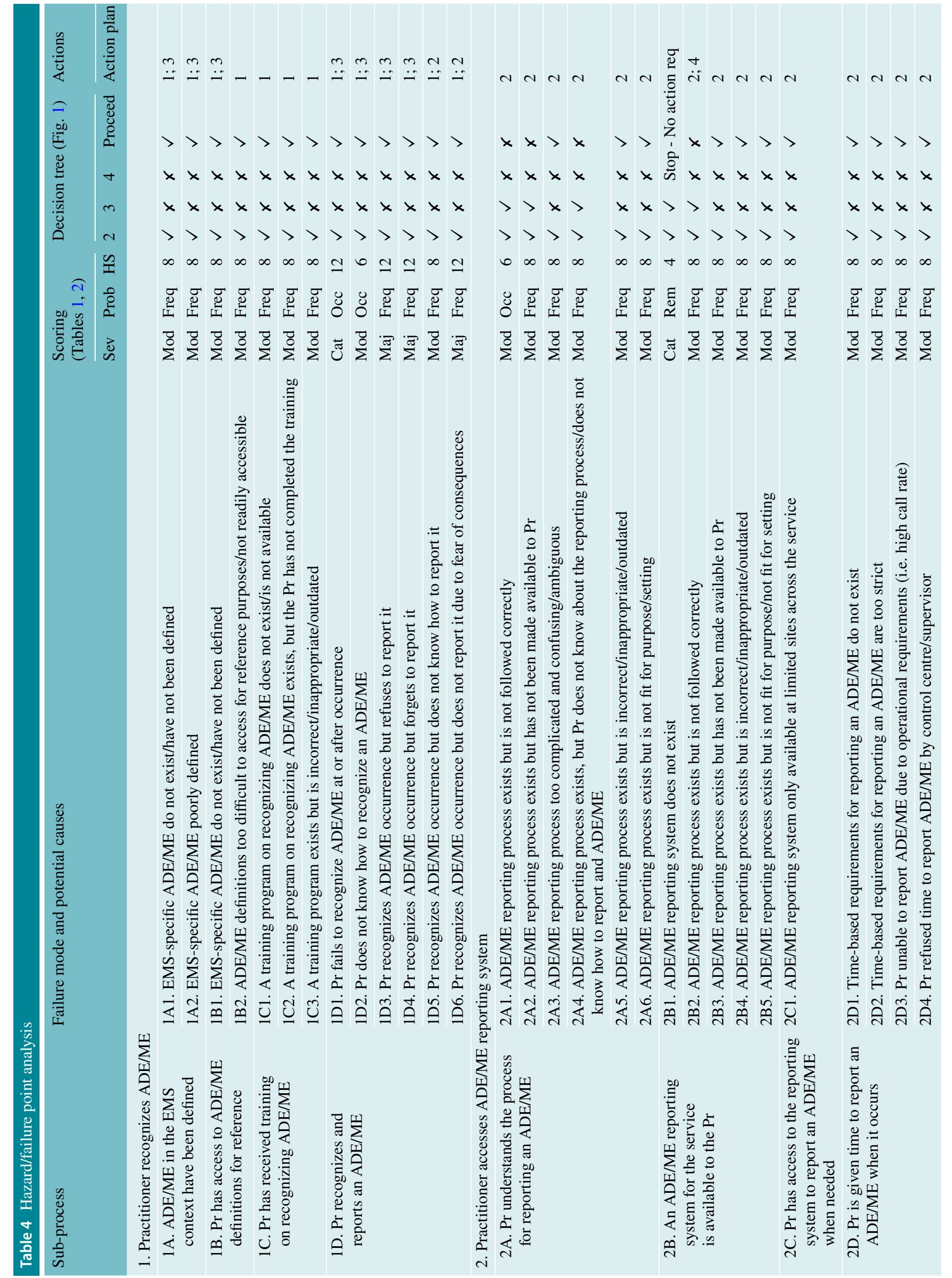




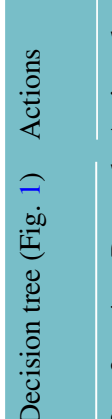

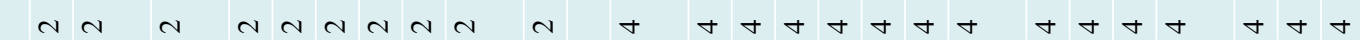

$x \times x \times x \times x \times x \times x \times x \times x \times x \times x$ $x \times x \times x \times x \times x \times x \times x \times x \times x \times x$ 岂 这

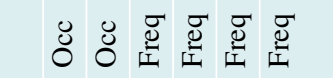

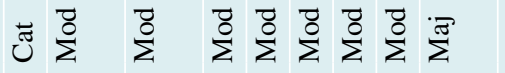

\section{8}

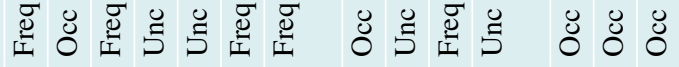

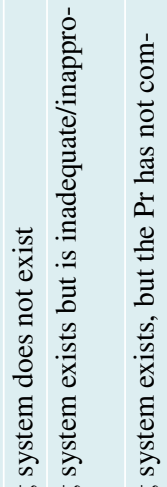

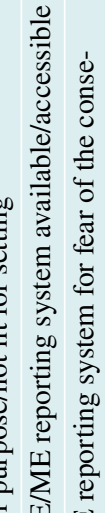

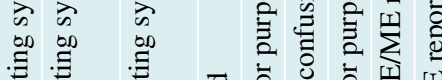

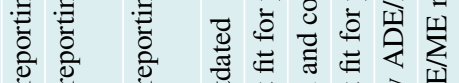

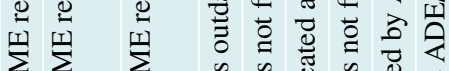

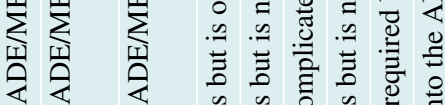

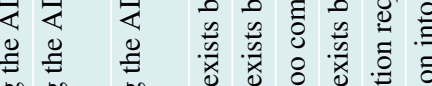

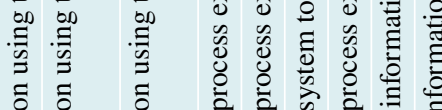
5 5 5 50

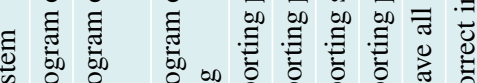

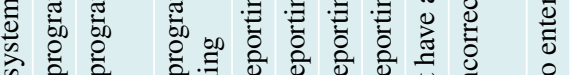

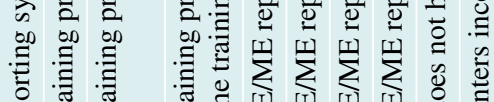

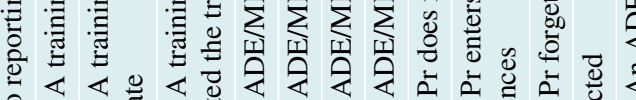

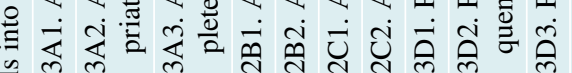
灵

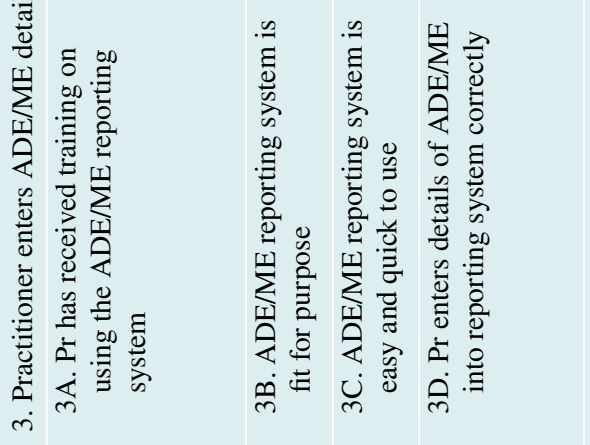

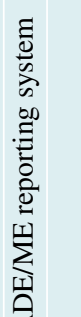

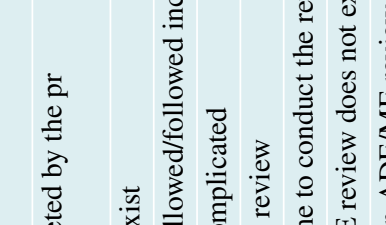

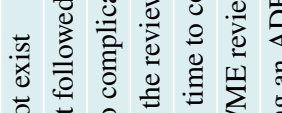

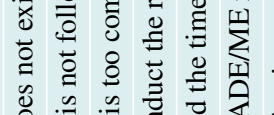

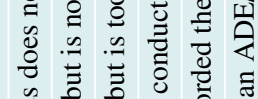

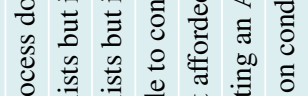

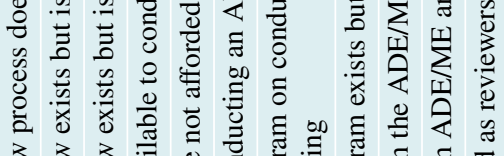

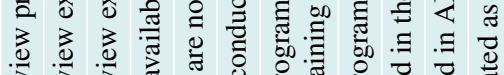

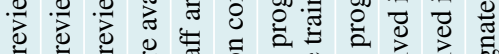

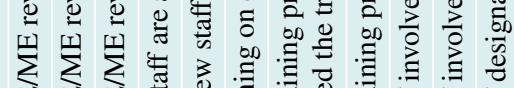

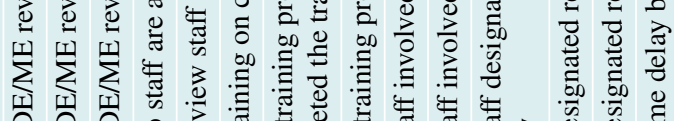

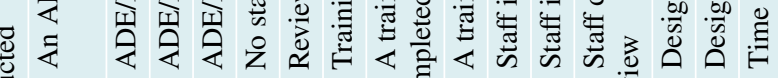

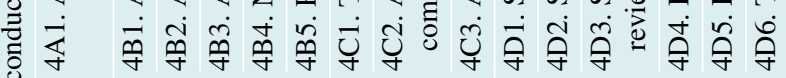

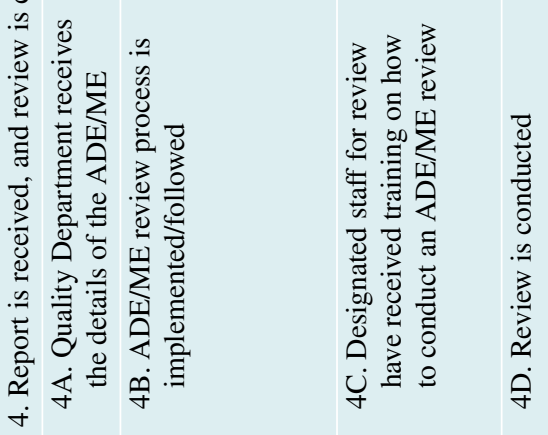




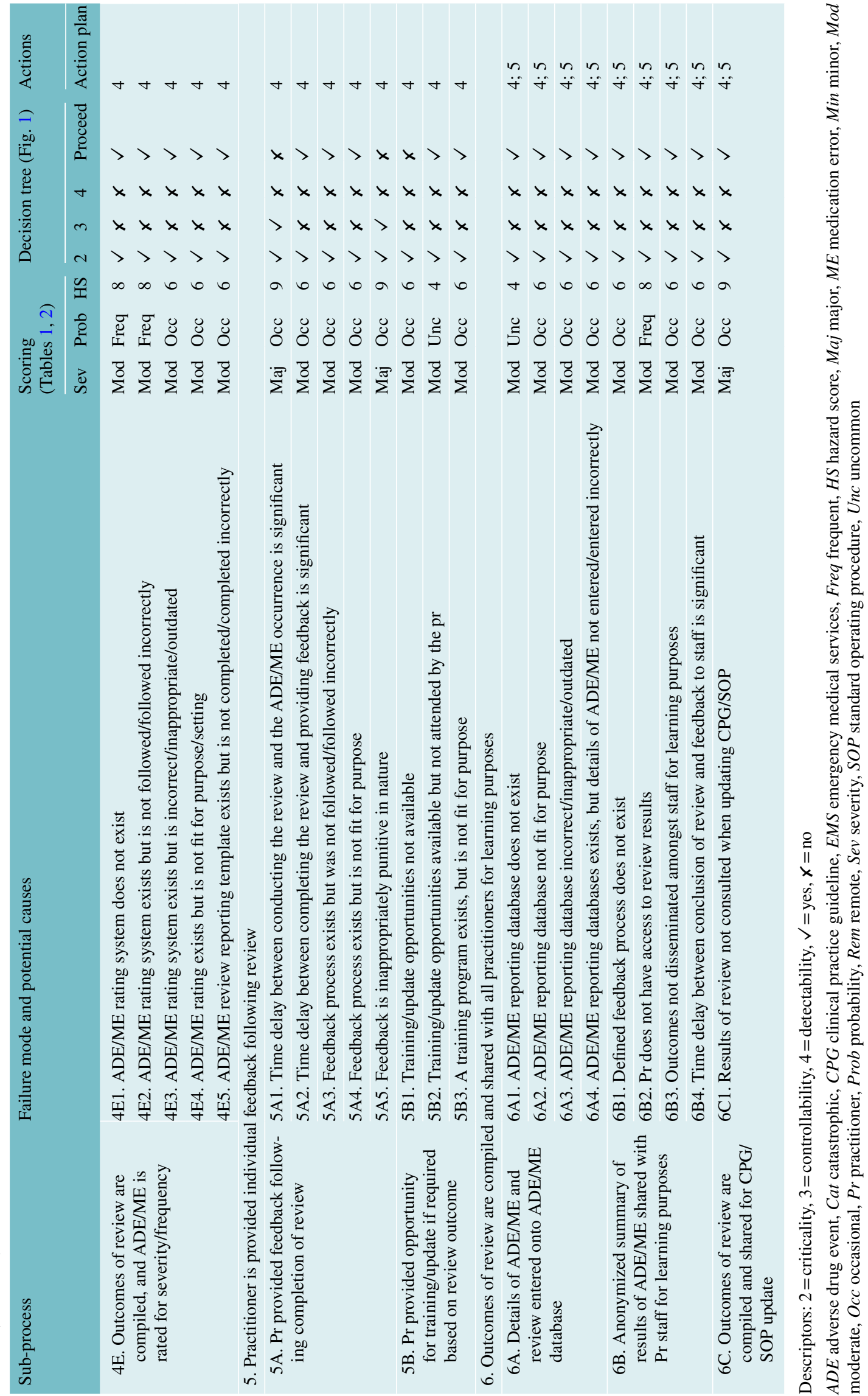


both reference and compliance. As a sub-point, these definitions could be included as part of a flagging system incorporated into an electronic patient care record (ePCR). This would potentially allow for increased 'realtime' detection of ADEs and MEs. A system analogous to the National Coordinating Council for Medication Error Reporting and Prevention (NCC MERP) categories, modified to the EMS context, was perceived to be the simplest yet most comprehensive towards achieving this [20,21].

- Action Plan 2 (AP2) Develop/implement/utilise an EMSspecific/fit-for-purpose reporting system that allows for the quick and simple reporting of ADEs and MEs. Given that EMS is a mobile service, fixed locations for reporting should be avoided, and reporting should ideally take advantage of mobile technology, potentially in the form of a smart phone application or incorporated into an ePCR. As a sub-point, time allowances for the reporting of ADEs and MEs by operational staff should be incorporated into formal operating procedures to provide opportunity for and encourage reporting. As a second sub-point, the option of anonymous reporting should be made available and/or included in the reporting process.

- Action Plan 3 (AP3) Develop/implement/utilise a reliable and validated tool to allow for the targeted detection of ADEs and MEs through an audit or governance process for the EMS setting. These should include standardised reporting measures and defined data-collection methods and data definitions. This would potentially allow for increased retrospective detection of missed cases not detected during or immediately after the delivery of care.

- Action Plan 4 (AP4) Develop/implement/utilise internal EMS staff resources dedicated to investigating ADEs and MEs. This should include allowing for dedicated and protected time for such staff to adequately and appropriately conduct an investigation should the need arise. As a subpoint, to ensure consistency, where possible, standardised templates for collecting and reporting information should be developed to aid the investigators' effort towards a final outcome.

- Action Plan 5 (AP5) A database of detected ADEs and MEs should be maintained to be used for training purposes and ideally incorporated into continued medical education sessions within an EMS organisation. As a sub-point, these should be periodically shared with frontline staff to allow for system transparency and continued professional education.

\section{Limitations}

The scope of this study was limited to the strategic-level focus on hazard/failure point identification, analysis and action plan development for system-focused factors affecting the reporting of ADEs and MEs in the EMS setting. No outcomes were measured or reported regarding the results of the actions identified in this study. The authors feel that a more intervention/experimental-focused research design may be more appropriate for such a study and, as such, remains an opportunity for future research. Furthermore, the examination of individual-focused influences to the identification and reporting of ADEs and MEs was beyond the scope of this study. The depth and breadth of such enquiry warrants its own individual study, and also remains an opportunity for future research.

\section{Discussion}

Following an annual audit of medication practices, it was determined that the rate of identification and reporting of ADEs and MEs within our service was lower than expected, a sentiment that was echoed in the literature. In a survey by Hobgood et al. $[8,22], 35 \%$ of participants had reported one to two medicationrelated errors in the previous 12 months, with an additional 9\% reporting more than two errors over the same time period. Of these, $89 \%$ were identified by the respondents themselves. The remaining errors were identified by patients, supervisors, nurses, fellow workers, or physicians prior to reporting. A total of $6 \%$ of errors identified by respondents were not reported [8, 22]. In a similar survey conducted by Vilke et al. [9], 9.1\% of respondents reported making an ME in the previous 12 months: $63 \%$ were dose-related errors, $33 \%$ were protocol errors, $21 \%$ were wrong route errors and $4 \%$ were wrong medication errors. The majority (79.1\%) were self-reported by respondents (79.1\%), with $8.3 \%$ being reported by the receiving hospital nurse; $4 \%$ of errors identified by respondents were not reported.

At face value, a lack of criteria for identifying MEs and ADEs would naturally seem to be the biggest barrier to their reporting, from a system point of view. However, once the entire process was mapped out, accessing an appropriate system to report the occurrence, and the subsequent steps necessary to conduct a review around the ADE/ME, were found to be the more complex constituents of this process. When potential failure points and/or hazards within this process were identified, these steps, in conjunction with the subprocesses required for providing feedback, were similarly found to not only possess the greatest number but also the majority requiring action to address and correct.

Based on the potential failure points and hazards identified in this study, several actions were identified that were found to be essential to prevent failure or mitigate the hazards within the ADE/ME identification and reporting process. The unique circumstances that occur within the out-of-hospital emergency care setting dictate that bespoke solutions be developed to allow for ease of implementation and ongoing success of use. This was evident in the five 
action plans identified within this study, which highlighted this point. Chief amongst this was the need for an EMS-specific system of identification. As comprehensive as systems such as the NCC MERP are, they remain too in-hospital focused for reliable and valid use in the out-of-hospital setting. Similarly, a reporting system that is mobile and/or takes advantage of mobile technology is crucial for a service that has mobility as one of its core functions.

The burden of ADEs in healthcare is an ever-increasing concern [23]. The potential for such events to occur within the out-of-hospital emergency care setting is significant. Despite this, there is a paucity of literature investigating ADEs in EMS, and, as such, little is known regarding their incidence. However, measuring and assessing these events is complex, largely due to their conceptual nature. ADEs are often intangible and therefore difficult to detect. Novel methodologies and approaches to their investigation and understanding are required to reduce their burden. This study represents one aspect of achieving this.

\section{Conclusion}

We mapped out a generic process for the detection, reporting and investigation of an ADE or ME in the out-of-hospital setting. Several potential system-focused points of failure and hazards were identified within this process. To counter these, and aid in the identification and reporting process, several action plans designed with the out-of-hospital setting in mind are essential to ensuring success.

Acknowledgements Open Access funding provided by the Qatar National Library.

\section{Compliance with ethical standards}

Conflict of interest Ian Howard, Nicholas Castle, Loua Al Shaikh, Ameena Jesaimani, and Robert Owen have no conflicts of interest that are directly relevant to the content of this article.

Informed consent All study participants received adequate information and agreed to participate before inclusion.

Funding No sources of funding were used to conduct this study or prepare this manuscript.

Ethical approval Ethical approval to conduct the study was granted by the Medical Research Centre of the Hamad Medical Corporation, Qatar.

Open Access This article is distributed under the terms of the Creative Commons Attribution-NonCommercial 4.0 International License (http://creativecommons.org/licenses/by-nc/4.0/), which permits any noncommercial use, distribution, and reproduction in any medium, provided you give appropriate credit to the original author(s) and the source, provide a link to the Creative Commons license, and indicate if changes were made.

\section{References}

1. Stirling CM, O'Meara P, Pedler D, et al. Engaging rural communities in health care through a paramedic expanded scope of practice. Rural Remote Health. 2007;7(4):839.

2. O'Meara PF, Tourle V, Stirling C, et al. Extending the paramedic role in rural Australia: a story of flexibility and innovation. Rural Remote Health. 2012;12(2):1978.

3. Woollard M. The role of the paramedic practitioner in the UK. J Emerg Prim Health Care. 2006;4(1):1-9.

4. Cooper S, Grant J. New and emerging roles in out of hospital emergency care: a review of the international literature. Int Emerg Nurs. 2009;17(2):90-8.

5. Al-Shaqsi S. Models of international emergency medical service (EMS) systems. Oman Med J. 2010;25(4):320-3.

6. Haupt MT, Bekes CE, Brilli RJ, et al. Guidelines on critical care services and personnel: Recommendations based on a system of categorization of three levels of care. Crit Care Med. 2003;31(11):2677-83.

7. Roudsari BS, Nathens AB, Cameron $P$, et al. International comparison of prehospital trauma care systems. Injury. 2007;38(9):993-1000.

8. Hobgood C, Bowen JB, Brice JH, et al. Do EMS personnel identify, report, and disclose medical errors? Prehospital Emerg Care. 2009;10(1):20-7.

9. Vilke GM, Tornabene SV, Stepanski B, et al. Paramedic self-reported medication errors. Prehospital Emerg Care. 2009;11(1):80-4.

10. Singh JM, Macdonald RD, Ahghari M, et al. Post-medication hypotension after administration of sedatives and opioids during critical care transport. Prehospital Emerg Care. 2015;19(4):464-74.

11. Dehours E, Bounes V, Bagheri H, et al. Adverse drug reactions in an emergency medical dispatching centre. Eur J Clin Pharmacol. 2014;70(7):881-7.

12. Lifshitz AE, Goldstein LH, Sharist M, et al. Medication prescribing errors in the prehospital setting and in the ED. Am J Emerg Med. 2012;30(5):726-31.

13. Institute for Safe Medication Practices. ISMP List of High-Alert Medications in Acute Care Settings [Internet]; 2018 [cited 2019 Jan 5]. https://www.ismp.org/sites/default/files/attachments/201810/highAlert2018new-Oct2018-v1.pdf.

14. Bahadori M, Smaeilnejad J, Sadeghifar J, et al. The factors affecting the refusal of reporting on medication errors from the nurses' viewpoints: a case study in a hospital in Iran. ISRN Nurs. 2013;2013:1-5.

15. Bayazidi S, Zarezadeh Y, Zamanzadeh V, et al. Medication error reporting rate and its barriers and facilitators among nurses. J Caring Sci. 2012;1(4):231-6.

16. Koohestani HR, Baghcheghi N. Barriers to the reporting of medication administration errors among nursing students. Aust $\mathbf{J}$ Adv Nurs. 2009;27(1):66-74.

17. Mirbaha F, Shalviri G, Yazdizadeh B, et al. Perceived barriers to reporting adverse drug events in hospitals: a qualitative study using theoretical domains framework approach. Implement Sci. 2015;10(1):1-10.

18. De Rosier J, Stalhandske E, Bagian J, et al. Using health care Failure Mode and Effect Analysis: the VA National Center for 
Patient Safety's prospective risk analysis system. Jt Comm J Qual Improv. 2002;28(5):248-67.

19. Shebl N, Franklin B, Barber N. Failure mode and effects analysis outputs: are they valid? BMC Health Serv Res. 2012;12:150.

20. Hartwig SC, Denger SD, Schneider PJ. Severity-indexed, incident report-based medication error-reporting program. Am J Health Pharm. 1991;48(12):2611-6.

21. National Coordinating Council for Medication Error Reporting and Prevention. NCC MERP index for categorizing medication errors algorithm; 2001. https://www.nccmerp.org/sites/default/ files/algorColor2001-06-12.pdf. Accessed 26 Mar 2019.

22. Hobgood C, Peck CR, Gilbert B, et al. Medical errors-what and when: what do patients want to know? Acad Emerg Med. 2002;9(11):1156-61.

23. Makary MA, Daniel M. Medical error: the third leading cause of death in the US. BMJ. 2016;353:i2139. 